\title{
Amplifying the Response of a Driven Resonator via Nonlinear Interaction with a Secondary Resonator
}

\section{Sahar Rosenberg ( $\nabla$ saharros@post.bgu.ac.il)}

Ben-Gurion University of the Negev https://orcid.org/0000-0001-6614-3782

\section{Oriel Shoshani}

Ben-Gurion University of the Negev

\section{Research Article}

Keywords: Nano- and micro-electro-mechanical-systems, Nonlinear driven resonators, Signal amplification

Posted Date: March 30th, 2021

DOl: https://doi.org/10.21203/rs.3.rs-328515/v1

License: (1) This work is licensed under a Creative Commons Attribution 4.0 International License. Read Full License

Version of Record: A version of this preprint was published at Nonlinear Dynamics on July 5th, 2021. See the published version at https://doi.org/10.1007/s11071-021-06659-x. 


\title{
Amplifying the response of a driven resonator via nonlinear interaction with a secondary resonator
}

\author{
Sahar Rosenberg* • Oriel Shoshani
}

Received: date / Accepted: date

\begin{abstract}
We study the dynamics of a resonantly driven nonlinear resonator (primary) that is nonlinearly coupled to a non-resonantly driven linear resonator (secondary) with a relatively short decay time. Due to its short relaxation time, the secondary resonator adiabatically tracks the primary resonator and modifies its response. Our model, which is motivated by experimental studies on the interaction between nano- and microresonators, is relatively simple and can be analyzed analytically and numerically to show that the driven response of the primary resonator can be enhanced significantly due to the interaction with the secondary resonator. Such an arrangement may pave the way for systematic control of driven responses and signal amplification in engineering applications involving nano- and micro-electro-mechanical-systems.
\end{abstract}

Keywords Nano- and micro-electro-mechanical-systems · Nonlinear driven resonators $\cdot$ Signal amplification

\section{Introduction}

Signal amplification of driven resonators is essential in engineering applications involving nano- and microelectro-mechanical-systems (N/MEMS). The importance of signal amplification stems from the need to operate above the noise-floor of the device with a large signal-to-noise ratio in order to use these tiny resonators for sensitive detection of displacement [1], mass [2], force [3], torque [4], and charge [5]. Due to its practical significance, signal amplification has received a considerable amount of attention [6-20]. Diverse methods have been devised for signal amplification including electrical means, such as lock-in amplifiers [21] and phase-locked loops [22], where the backaction from the inherently noisy electrical amplifier needs to be treated, and mechanical means, where the motion of the resonator is mechanically preamplified by a large factor before being transduced into an electrical signal.

The realm of mechanical amplification is vast and includes numerous distinguishable techniques, such as bifurcation-topology amplification [9], strong mechanical coupling amplification [12], and parametric amplification [7]. The latter, i.e., parametric amplification - the process of amplifying a driven resonator with a parametric pump at twice its oscillation frequency and with a phase-delay, is a well established concept and the most widely used technique with applications ranging from radio engineering [23] to cavity optomechanics [24].

In this study we present a relatively simple amplification scheme in which a pair of driven mechanical resonators are mutually coupled. One resonator (the secondary) decays much faster than the primary resonator and can serve as an amplifier for the primary resonator. The amplification of the primary resonator depends on the coupling between the resonators and the driving frequency of the secondary resonator. Specifically, when the coupling is linear and the driving frequency of the secondary resonator is close to the eigenfrequency of the primary resonator, a significant enhancement in the drive level of the primary resonator is obtained, and when the coupling is nonlinear (quadratic) and the driving frequency of the secondary resonator is close to twice the eigenfrequency of the primary resonator, a parametric amplification is obtained.

Our amplification technique comes from a straightforward generalization of a previous study that was conducted on the undriven counterpart of the dynamical system under consideration [25]. The previous study, Ref. [25], showed that in the undriven system, the linear and nonlinear characteristics of the primary resonator can

S. Rosenberg (Corresponding author)

Ben-Gurion University of the Negev, Beer-Sheva 84105, Israel

E-mail: saharros@post.bgu.ac.il

O. Shoshani

Ben-Gurion University of the Negev, Beer-Sheva 84105, Israel

E-mail: oriels@bgu.ac.il 
be altered in a significant manner. Therefore, as we show in the present analysis, in the driven system it is possible to both modify the characteristics of the primary resonator and amplify its response.

This paper is organized as follows: In Sect. 2 we formulate the problem and show that the motion of the relatively fast decaying secondary resonator can be eliminated from the governing equation of the primary resonator under the adiabatic approximation for time that are considerably larger then the relaxation time of the secondary resonator. In Sect. 3 we conduct an asymptotic analysis and obtain the main results of the paper, consisting of closed-form expressions for the amplified response of the primaryresonator. In addition, we present numerical validation of our theoretical predictions. Finally, in Sect. 4 we summarize our main findings, discuss their implications, and suggest potential future work.

\section{Problem formulation}

We consider a system consisting of a pair of driven resonators that are mutually coupled (Fig. 1). The primary

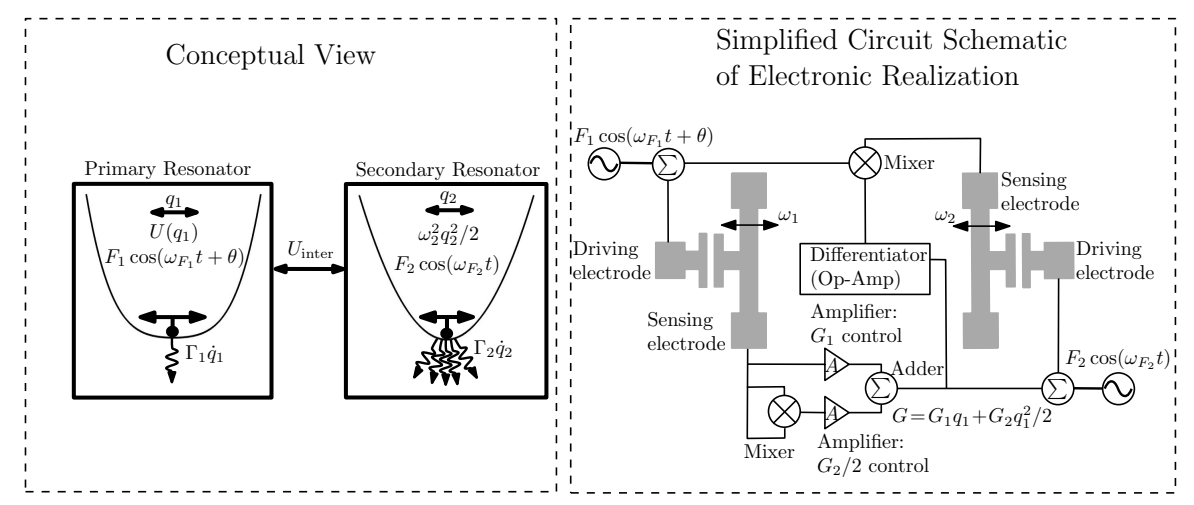

Fig. 1 Conceptual view and simplified circuit schematic of an electronic realization. Left panel: Two resonators are mutually coupled via the interaction potential $-q_{2} G\left(q_{1}\right)$. The primary resonator $q_{1}$ has a nonlinear conservative restoring force, $-U^{\prime}\left(q_{1}\right)$, a weak linear dissipation $-\Gamma_{1} \dot{q}_{1}$, and a resonant drive $F_{1} \cos \left(\omega_{F_{1}} t+\theta\right)$, where $\omega_{F_{1}} \approx \sqrt{U^{\prime \prime}(0)}$. The secondary resonator has a linear conservative restoring force $-\omega_{2}^{2} q_{2}$, a relatively strong linear dissipation $-\Gamma_{2} \dot{q}_{2}$, and a non-resonant drive $F_{2}$ cos $\left(\omega_{F_{2}} t\right)$. The dissipation is indicated by the wiggly lines, which indicates that the secondary resonator is more heavily damped than the primary resonator. Right panel: For N/MEMS, the motion of each resonator is detected using a parallel-plate capacitive sensing technique. The signal from the primary resonator is fed into a mixer, amplifiers (for control of the different coefficients of $G$ ), and an adder to form the function $G\left(q_{1}\right)$. The signal $G\left(q_{1}\right)$ is then fed into the driving electrode of the secondary resonator along with the external drive $F_{2} \cos \left(\omega_{F_{2}} t\right)$, and to a differentiator and a mixer, which multiplies the signal $G^{\prime}\left(q_{1}\right)$ with $q_{2}$, and finally, the mixed signal $q_{2} G^{\prime}\left(q_{1}\right)$ is fed into the driving electrode of the primary resonator along with the external drive $F_{1} \cos \left(\omega_{F_{1}} t+\theta\right)$.

resonator $q_{1}$ is driven resonantly by an excitation $F_{1} \cos \left(\omega_{F_{1}} t+\theta\right)$ and oscillates around its stable equilibrium $q_{1}=0$ with a large amplitude and nonlinear restoring forces that stem from a potential $U\left(q_{1}\right)$. The secondary resonator $q_{2}$ is driven by a non-resonant excitation $F_{2} \cos \left(\omega_{F_{2}} t\right)$, i.e., the drive frequency $\omega_{F_{2}}$ is not close to its eigenfrequency $\omega_{2}$, and oscillates linearly with small amplitude around its stable equilibrium $q_{2}=0$. The two resonators are coupled via an interaction potential $U_{\text {inter }}\left(q_{1}, q_{2}\right)$. Since $\left|q_{1}\right| \gg\left|q_{2}\right|$, we assume that the interaction potential depends nonlinearly on $q_{1}$ and linearly on $q_{2}$, i.e., $U_{\text {inter }}=-q_{2} G\left(q_{1}\right)$. Therefore, the Hamiltonian of the system is given by

$$
\mathcal{H}=p_{1}^{2} / 2+p_{2}^{2} / 2+U\left(q_{1}\right)+\omega_{2}^{2} q_{2}^{2} / 2-q_{2} G\left(q_{1}\right)-q_{1} F_{1} \cos \left(\omega_{F_{1}} t+\theta\right)-q_{2} F_{2} \cos \left(\omega_{F_{2}} t\right)
$$

The governing equations of $q_{1}$ and $q_{2}$ are formally given by $\ddot{q}_{k}=\dot{p}_{k}=-2 \Gamma_{k} \dot{q}_{k}-\partial \mathcal{H} / \partial q_{k}$, and can be explicitly written as

$$
\begin{aligned}
& \ddot{q}_{1}=-2 \Gamma_{1} \dot{q}_{1}-U^{\prime}\left(q_{1}\right)+F_{1} \cos \left(\omega_{F_{1}} t+\theta\right)+q_{2} G^{\prime}\left(q_{1}\right), \\
& \ddot{q}_{2}=-2 \Gamma_{2} \dot{q}_{2}-\omega_{2}^{2} q_{2}+F_{2} \cos \left(\omega_{F_{2}} t\right)+G\left(q_{1}\right),
\end{aligned}
$$

where the dot symbol denotes a time derivative, the prime symbol denotes a differentiation of a function with respect to its single variable, and $-2 \Gamma_{k} \dot{q}_{k}$ models the linear friction force experienced by resonator $k(k=1,2)$. Moreover, both resonators are lightly damped $\left(\Gamma_{k} / \omega_{k} \ll 1\right)$, and the secondary resonator decays much faster than the primary resonator $\left(\Gamma_{2} / \Gamma_{1} \gg 1\right)$. 
Eq. (3) is linear in $q_{2}$, therefore, we can formally solve for $q_{2}$ in terms of its Green's function

$$
\begin{aligned}
q_{2}(t)= & e^{-\Gamma_{2} t}\left[q_{2}(0) \cos \left(\omega_{2 d} t\right)+\frac{p_{2}(0)}{\omega_{2 d}} \sin \left(\omega_{2 d} t\right)\right] \\
& +\frac{1}{\omega_{2 d}} \int_{0}^{t}\left[F_{2} \cos \left(\omega_{F_{2}} \tau\right)+G\left(q_{1}(\tau)\right)\right] e^{-\Gamma_{2}(t-\tau)} \sin \left(\omega_{2 d}(t-\tau)\right) d \tau .
\end{aligned}
$$

Because we consider a lightly damped system $\left(\Gamma_{k} / \omega_{k} \ll 1\right)$, the damped eigenfrequency of the secondary resonator in Eq. (4) can be replaced by the undamped eigenfrequency, i.e., $\omega_{2 d}=\omega_{2} \sqrt{1-\left(\Gamma_{2} / \omega_{2}\right)^{2}} \approx \omega_{2}$. By substitution of Eq. (4) into Eq. (2) we find that

$$
\ddot{q}_{1}=-2 \Gamma_{1} \dot{q}_{1}-U^{\prime}\left(q_{1}\right)+F_{1} \cos \left(\omega_{F_{1}} t+\theta\right)+G^{\prime}\left(q_{1}\right)\left(L\left[q_{1}, t\right]+M(t)\right),
$$

where

$$
\begin{aligned}
L\left[q_{1}, t\right] & =\frac{1}{\omega_{2}} \int_{0}^{t}\left[F_{2} \cos \left(\omega_{F_{2}} \tau\right)+G\left(q_{1}(\tau)\right)\right] e^{-\Gamma_{2}(t-\tau)} \sin \left(\omega_{2}(t-\tau)\right) d \tau, \\
M(t) & =e^{-\Gamma_{2} t}\left[q_{2}(0) \cos \left(\omega_{2} t\right)+\frac{p_{2}(0)}{\omega_{2}} \sin \left(\omega_{2} t\right)\right] .
\end{aligned}
$$

The functional $L\left[q_{1}, t\right]$ is the driven response (by the external excitation and the primary resonator) of the secondary, and $M(t)$ is the response of the secondary resonator to its initial state.

\section{Asymptotic analysis}

To analyze Eq. (5) we make a number of standard assumptions that are relevant to weakly nonlinear resonators, i.e., that all effects other than the inertia $\left(\ddot{q}_{1}\right)$ and linear stiffness $\left[U^{\prime \prime}(0) q_{1}\right]$ of resonator are small. Specifically, we assume a weak nonlinear interaction between the resonators $\left[G^{\prime}\left(q_{1}\right)\left(L\left[q_{1}, t\right]+M(t)\right) /\left|U^{\prime \prime}(0) q_{1}\right| \ll 1\right]$, a weak damping $\left(\left|2 \Gamma_{1} \dot{q}_{1}\right| /\left|U^{\prime \prime}(0) q_{1}\right| \ll 1\right)$, and a weak drive of the primary resonator $\left(F_{1} /\left|U^{\prime \prime}(0) q_{1}\right| \ll 1\right)$. Under these assumptions we can use the method of averaging to derive evolution equations for the slow variations of the amplitude and phase of the primary resonator $[26,27]$.

To this end, we make the following coordinate transformation $q_{1}(t)=\mathcal{A}(t) e^{i \omega_{F_{1}} t}+\mathcal{A}^{*}(t) e^{-i \omega_{F_{1}} t}$ and $\dot{q}_{1}(t)=$ $i \omega_{F_{1}}\left[\mathcal{A}(t) e^{i \omega_{F_{1}} t}-\mathcal{A}^{*}(t) e^{-i \omega_{F_{1}} t}\right]$, where $\mathcal{A}^{*}$ is the complex-conjugate of $\mathcal{A}$, and the complex amplitude $\mathcal{A}(t)$, remains almost constant over the period $T=2 \pi / \omega_{F_{1}}$. Consequently, $G\left(q_{1}(t)\right)$ can be replaced by its Fourier series $G\left(q_{1}(t)\right)=\sum_{n} c_{n}\left(\mathcal{A}(t), \mathcal{A}^{*}(t)\right) e^{i n \omega_{F_{1}} t}$, where $c_{-n}=c_{n}^{*}$, and the functional, $L\left[q_{1}, t\right]$, can be rewritten as

$$
\begin{aligned}
L\left[q_{1}, t\right]=\frac{F_{2}}{\omega_{2}} \int_{0}^{t} e^{-\Gamma_{2}(t-\tau)} \sin \left(\omega_{2}(t-\tau)\right) \cos \left(\omega_{F_{2}} \tau\right) d \tau \\
\quad+\int_{0}^{t} \sum_{n} \frac{c_{n}(\tau)}{2 i \omega_{2}}\left[e^{-\Gamma_{2}(t-\tau)+i\left[n \omega_{F_{1}} \tau+\omega_{2}(t-\tau)\right]}-e^{-\Gamma_{2}(t-\tau)+i\left[n \omega_{F_{1}} \tau-\omega_{2}(t-\tau)\right]}\right] d \tau
\end{aligned}
$$

Because $c_{n}(t)$ are functions of $\mathcal{A}(t)$ and $\mathcal{A}^{*}(t)$, they also vary slowly in time. Hence, to the leading order, we can approximate Eq. (8) in the following way

$$
\begin{aligned}
L\left[q_{1}, t\right] \approx & \frac{F_{2}}{\omega_{2}} \int_{0}^{t} e^{-\Gamma_{2}(t-\tau)} \sin \left(\omega_{2}(t-\tau)\right) \cos \left(\omega_{F_{2}} \tau\right) d \tau \\
& +\sum_{n} \frac{c_{n}(t)}{2 i \omega_{2}} \int_{0}^{t}\left[e^{-\Gamma_{2}(t-\tau)+i\left[n \omega_{F_{1}} \tau+\omega_{2}(t-\tau)\right]}-e^{-\Gamma_{2}(t-\tau)+i\left[n \omega_{F_{1}} \tau-\omega_{2}(t-\tau)\right]}\right] d \tau \\
= & F_{2}\left[k_{1} \cos \left(\omega_{F_{2}} t-\varphi\right)-k_{2} e^{-\Gamma_{2} t} \cos \left(\omega_{F_{2}} t-\psi\right)\right] \\
& +\sum_{n} \frac{c_{n}(t)}{2 \omega_{2}} e^{i n \omega_{F_{1}} t}\left[\frac{1-e^{-\left[\Gamma_{2}+i\left(n \omega_{F_{1}}-\omega_{2}\right)\right] t}}{-\left(n \omega_{F_{1}}-\omega_{2}\right)+i \Gamma_{2}}+\frac{1-e^{-\left[\Gamma_{2}+i\left(n \omega_{F_{1}}+\omega_{2}\right)\right] t}}{\left(n \omega_{F_{1}}+\omega_{2}\right)-i \Gamma_{2}}\right]
\end{aligned}
$$

where

$$
\begin{array}{ll}
k_{1}=\frac{\sqrt{\left(\omega_{2}^{2}-\omega_{F_{2}}^{2}+\Gamma_{2}^{2}\right)^{2}+\left(2 \omega_{F_{2}} \Gamma_{2}\right)^{2}}}{\left[\left(\omega_{2}-\omega_{F_{2}}\right)^{2}+\Gamma_{2}^{2}\right]\left[\left(\omega_{2}+\omega_{F_{2}}\right)^{2}+\Gamma_{2}^{2}\right]}, & \tan \varphi=\frac{2 \omega_{F_{2}} \Gamma_{2}}{\omega_{2}^{2}-\omega_{F_{2}}^{2}+\Gamma_{2}^{2}} \\
k_{2}=\frac{\sqrt{\left(\omega_{2}^{2}-\omega_{F_{2}}^{2}+\Gamma_{2}^{2}\right)^{2}+\left(\Gamma_{2} / \omega_{2}\right)^{2}\left(\omega_{2}^{2}+\omega_{F_{2}}^{2}+\Gamma_{2}^{2}\right)^{2}}}{\left[\left(\omega_{2}-\omega_{F_{2}}\right)^{2}+\Gamma_{2}^{2}\right]\left[\left(\omega_{2}+\omega_{F_{2}}\right)^{2}+\Gamma_{2}^{2}\right]}, \tan \psi=\frac{\Gamma_{2}}{\omega_{2}^{2}+\omega_{F_{2}}^{2}+\Gamma_{2}^{2}} \frac{\omega_{2}^{2}-\omega_{F_{2}}^{2}+\Gamma_{2}^{2}}{} .
\end{array}
$$


We note that for $t \gg \Gamma_{2}^{-1}$, the functional $L\left[q_{1}, t\right]$ is simplified considerably and reduces to

$$
L\left[q_{1}, t\right] \approx k_{1} F_{2} \cos \left(\omega_{F_{2}} t-\varphi\right)+\sum_{n} \frac{\omega_{2}^{2}-n^{2} \omega_{F_{1}}^{2}-2 i n \omega_{F_{1}} \Gamma_{2}}{\left(\omega_{2}^{2}-n^{2} \omega_{F_{1}}^{2}\right)^{2}+\left(2 n \omega_{F_{1}} \Gamma_{2}\right)^{2}} c_{n}(t) e^{i n \omega_{F_{1}} t}
$$

Hence, we effectively eliminated the secondary resonator and obtained from Eq. (5) the following evolution equation of the primary resonator

$$
\begin{aligned}
\ddot{q}_{1}+U^{\prime}\left(q_{1}\right)=F_{1} \cos \left(\omega_{F_{1}} t+\theta\right)+k_{1} G^{\prime}\left(q_{1}\right) F_{2} \cos \left(\omega_{F_{2}} t-\varphi\right) & \\
& -2 \Gamma_{1} \dot{q}_{1}+G^{\prime}\left(q_{1}\right) \sum_{n} \frac{\omega_{2}^{2}-n^{2} \omega_{F_{1}}^{2}-2 i n \omega_{F_{1}} \Gamma_{2}}{\left(\omega_{2}^{2}-n^{2} \omega_{F_{1}}^{2}\right)^{2}+\left(2 n \omega_{F_{1}} \Gamma_{2}\right)^{2}} c_{n}(t) e^{i n \omega_{F_{1}} t} .
\end{aligned}
$$

Moreover, this approximation applies even for internal-resonance situations, where $\omega_{2}$ is close to a multiple of $\omega_{1}$.

In the remainder of this paper, we focus only on times that are considerably larger then the relaxation time of the secondary resonator $t \gg \Gamma_{2}^{-1}$, where the secondary resonator is adiabatically tracking the primary resonator [28]. We have also assumed here that the functions $c_{n}(t)$ vary slowly over the time $\Gamma_{2}^{-1}$, which is a restriction on the nonlinearity and the decay rate of the primary resonator. For the weak coupling that we consider here, the only terms to keep in the sum over $n$ of Eq. (12) are the resonant terms, which oscillate with slowly varying amplitude and phase at the same frequency as $q_{1}(t)$. For linear coupling, where $G\left(q_{1}\right) \propto q_{1}$ and $G^{\prime}\left(q_{1}\right)$ is constant, the resonant terms are associated with the fundamental harmonic $(n= \pm 1)$, whereas for quadratic coupling in which $G\left(q_{1}\right) \propto q_{1}^{2}$ and $G^{\prime}\left(q_{1}\right)$ is a linear function of $q_{1}$, we have to keep the second $(n= \pm 2)$ and zeroth $(n=0)$ harmonics. The same arguments hold for the external excitation of the secondary resonator $F_{2} \cos \left(\omega_{F_{2}} t\right)$, i.e., for constant $G^{\prime}$, only a drive frequency $\omega_{F_{2}} \approx \omega_{1}$ will lead to a resonant term ${ }^{1}$, and if we take into account the term $\propto q_{1}$ in $G^{\prime}\left(q_{1}\right)$, only a drive frequency $\omega_{F_{2}} \approx 2 \omega_{1}$ will lead to a resonant term. These distinctions are used in the analysis of the next subsections.

\subsection{Essential leading-order nonlinear terms}

In what follows we restrict our attention to the essential leading-order nonlinear terms. We assume that both $U\left(q_{1}\right)$ and $G\left(q_{1}\right)$ are analytic functions, and hence, can be expanded to a Taylor series. Thus, the potential of the primary resonator can be explicitly written as a Taylor expansion truncated at quartic order $U\left(q_{1}\right)=$ $\omega_{1}^{2} q_{1}^{2} / 2+\gamma q_{1}^{4} / 4$, where the non-secular term $\beta q_{1}^{3} / 3$ renormalizes $\gamma$ to the second order in $\beta$, which we assume to have been taken into account. Similarly, $G\left(q_{1}\right)$ can represented as $G\left(q_{1}\right)=G_{1} q_{1}+G_{2} q_{1}^{2} / 2+O\left(q_{1}^{3}\right)$, where $G_{1} \equiv G^{\prime}(0)$ and $G_{2} \equiv G^{\prime \prime}(0)$. Therefore, we obtain the following complex amplitude equation of the primary resonator

$$
\begin{aligned}
\dot{\mathcal{A}}= & -\Gamma_{1} \mathcal{A}\left(1+\alpha_{1} \frac{\Gamma_{2}}{\Gamma_{1}}+\alpha_{2} \frac{\Gamma_{2}}{\Gamma_{1}}|\mathcal{A}|^{2}\right) \\
& -i \mathcal{A}\left\{\delta \omega_{1}+\alpha_{1}\left(\frac{\omega_{2}^{2}-\omega_{1}^{2}}{2 \omega_{1}}\right)+\left[\alpha_{2}\left(\frac{3 \omega_{2}^{2}-20 \omega_{1}^{2}}{4 \omega_{1}}+\frac{8 \omega_{1}}{\omega_{2}^{2}}\left(\Gamma_{2}^{2}+\omega_{1}^{2}\right)\right)-\frac{3 \gamma}{2 \omega_{1}}\right]|\mathcal{A}|^{2}\right\} \\
& -\frac{i}{4 \omega_{1}}\left(F_{1} \exp (i \theta)+k_{1} G_{1} F_{2} \exp \left\{i\left[\left(\omega_{F_{2}}-\omega_{F_{1}}\right) t-\varphi\right]\right\}+k_{1} G_{2} \mathcal{A}^{*} F_{2} \exp \left\{i\left[\left(\omega_{F_{2}}-2 \omega_{F_{1}}\right) t-\varphi\right]\right\}\right),
\end{aligned}
$$

where we replaced $\omega_{F_{1}}$ with $\omega_{1}$ in the various coefficients of Eq. (13) other than the detuning parameter $\delta \omega_{1}=\omega_{F_{1}}-\omega_{1}$, since it leads to higher corrections that can be ignored at the current level of approximation. Accordingly, the coupling to the secondary resonator is captured by the terms with the coefficients

$$
\alpha_{1}=\frac{G_{1}^{2}}{\left(\omega_{2}^{2}-\omega_{1}^{2}\right)^{2}+\left(2 \Gamma_{2} \omega_{1}\right)^{2}}, \quad \alpha_{2}=\frac{G_{2}^{2}}{\left(\omega_{2}^{2}-4 \omega_{1}^{2}\right)^{2}+\left(4 \Gamma_{2} \omega_{1}\right)^{2}} .
$$

Hence, as shown in Ref. [25], the interaction of the primary resonator with the secondary resonator modifies the linear and nonlinear restoring forces of the primary resonator and leads to:

1. An increase in the linear decay rate $\Gamma_{1 \text { eff }}=\Gamma_{1}+\alpha_{1} \Gamma_{2}$

2. Addition of new nonlinear cubic (van der Pol type) damping term $-\alpha_{2} \Gamma_{2}|\mathcal{A}|^{2} \mathcal{A}$

3. A shift in the eigenfrequency $\Delta \omega_{1}=\alpha_{1}\left(\omega_{2}^{2}-\omega_{1}^{2}\right) /\left(2 \omega_{1}\right)$

4. A modification to the Duffing nonlinearity $-i \alpha_{2}\left[\left(3 \omega_{2}^{2}-20 \omega_{1}^{2}\right) /\left(4 \omega_{1}\right)+8 \omega_{1}\left(\Gamma_{2}^{2}+\omega_{1}^{2}\right) / \omega_{2}^{2}\right]|\mathcal{A}|^{2} \mathcal{A}$

1 Under the assumption of weak excitation, where $\left|U^{\prime \prime}(0) q_{1}\right| \gg k_{1} G^{\prime} F_{2}$. 
Moreover, in the current setup of driven resonators, the interaction of the primary resonator with the secondary resonator also modifies the external drive. In particular, there are two cases that lead to additional resonant driving force:

- Case I: where $\omega_{F_{2}}=\omega_{F_{1}}$, and the uncoupled direct external drive term $F_{1} e^{i \theta}$ in Eq. (13) is replaced by $F_{1} e^{i \theta}+k_{1} G_{1} F_{2} e^{-i \varphi}$, which modifies both the amplitude and phase of the direct external drive. The parametric drive in Eq. (13), $k_{1} G_{2} \mathcal{A}^{*} F_{2} e^{-i\left(\omega_{F_{1}} t+\varphi\right)}$, is averaged to zero. Hence, in this case, Eq. (13) is consistent with the complex amplitude equation that is obtained from the averaging method for the following phenomenological model

$$
\ddot{q}_{1}+2\left(\Gamma_{1 \mathrm{eff}} \dot{q}_{1}+\alpha_{2} \Gamma_{2} q_{1}^{2}\right) \dot{q}_{1}+\tilde{\omega}_{1}^{2} q_{1}+\tilde{\gamma} q_{1}^{3}=\tilde{F} \cos \left(\omega_{F_{1}} t+\chi\right)
$$

where the square of the shifted eigenfrequency is given by $\tilde{\omega}_{1}^{2}=\omega_{1}^{2}-\alpha_{1}\left(\omega_{2}^{2}-\omega_{1}^{2}\right)$, the modified Duffing parameter is given by $\tilde{\gamma}=\gamma-\left(2 \alpha_{2} / 3\right)\left[\left(3 \omega_{2}^{2}-20 \omega_{1}^{2}\right) / 4+8 \omega_{1}^{2}\left(\Gamma_{2}^{2}+\omega_{1}^{2}\right) / \omega_{2}^{2}\right]$, the modified amplitude and phase of the direct drive in Eq. (15) are given by $F=\sqrt{F_{1}^{2}+2 k_{1} G_{1} F_{1} F_{2} \cos (\theta+\varphi)+k_{1}^{2} G_{1}^{2} F_{2}^{2}}$ and $\tan \chi=\left(F_{1} \sin \theta-k_{1} G_{1} F_{2} \sin \varphi\right) /\left(F_{1} \cos \theta+k_{1} G_{1} F_{2} \cos \varphi\right)$.

- Case II: where $\omega_{F_{2}}=2 \omega_{F_{1}}$, and the direct external drive $F_{1} e^{i \theta}$ remains unmodified because the term $k_{1} G_{1} F_{2} e^{i\left(\omega_{F_{1}} t-\varphi\right)}$ is averaged to zero; however, there is an additional parametric drive term $k_{1} G_{2} \mathcal{A}^{*} F_{2} e^{-i \varphi}$ that can lead to non-trivial dynamical outcomes. In this case, Eq. (13) is consistent with the complex amplitude equation that is obtained from the averaging method for the following phenomenological model

$$
\ddot{q}_{1}+2\left(\Gamma_{1 \mathrm{eff}} \dot{q}_{1}+\alpha_{2} \Gamma_{2} q_{1}^{2}\right) \dot{q}_{1}+\left[\tilde{\omega}_{1}^{2}-k_{1} G_{2} F_{2} \cos \left(\omega_{F_{2}} t-\varphi\right)\right] q_{1}+\tilde{\gamma} q_{1}^{3}=F_{1} \cos \left(\omega_{F_{1}} t+\theta\right) .
$$

These two cases are considered in detail in the following subsections.

\subsection{The case of $\omega_{F_{2}}=\omega_{F_{1}}$}

While the modified linear $\left(\tilde{\omega}_{1}, \Gamma_{1 \text { eff }}\right)$ and nonlinear $\left(\alpha_{2} \Gamma_{2}, \tilde{\gamma}\right)$ characteristics of the primary resonator have been discussed in Ref. [25], the variation of the drive amplitude $\tilde{F}$ and phase $\chi$ are new phenomena that deserve further attention. We focus here on the dynamical systems, where in addition to the large difference in the relaxation times $\Gamma_{2} / \Gamma_{1} \gg 1$, there is a large difference in resonators eigenfrequencies $\omega_{2} / \omega_{1} \gg 1$. These conditions are frequently occur in the nonlinear interactions between MEMS (primary) resonators and NEMS (secondary) resonators [29-31]. From Fig. 2 we see that for a finite frequency ratio $\omega_{2} / \omega_{1}$, there is a major enhancement
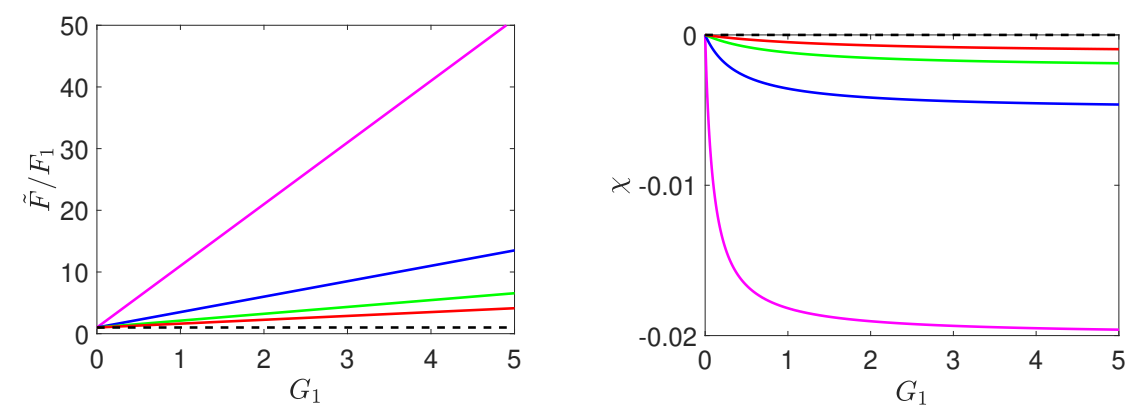

Fig. 2 Variation of the direct drive normalized amplitude $\left(\tilde{F} / F_{1}\right)$ and phase $(\chi)$ of the primary resonator as a function of the linear coupling to the secondary resonator $\left(G_{1}\right)$ for $\Gamma_{2} / \Gamma_{1}=F_{2} / F_{1}=10^{3}, \theta=0$, and different frequency ratios: $\omega_{2} / \omega_{1}=10($ magenta), $\omega_{2} / \omega_{1}=20$ (blue), $\omega_{2} / \omega_{1}=30$ (green), $\omega_{2} / \omega_{1}=40$ (red), and $\omega_{2} / \omega_{1} \rightarrow \infty$ (dashed black), which is equivalent to the case of no linear coupling $\left(G_{1}=0\right)$. Left panel: The normalized drive amplitude increases significantly as the frequency ratio decreases. Right panel: The phase of the direct drive undergoes an insignificant variation as long as the eigenfrequency of the secondary resonator is an order of magnitude higher than the eigenfrequency of the primary resonator.

of the drive amplitude due to the coupling with the secondary resonator (e.g., $\tilde{F} \approx 50 F$ for $\omega_{2} / \omega_{1}=10$ and $G_{1}=4.9$ ), and a minor variation of the phase (e.g., $\chi \approx-0.02$ for $\omega_{2} / \omega_{1}=10$ and $\left.G_{1}=4.9\right)$. These results suggest that we can use the linear coupling of the resonators $G_{1}$ to amplify the response of the primary resonator.

To further understand how the interaction with the secondary resonator changes the forced response of the primary resonator, we write the complex amplitude in polar representation $\mathcal{A}=a e^{i \phi} / 2$, and consider the evolution of the amplitude $a$ and phase $\phi$

$$
\begin{aligned}
& \dot{a}=-\left(\Gamma_{1 \mathrm{eff}}+\frac{\alpha_{2} \Gamma_{2} a^{2}}{4}\right) a+\frac{\tilde{F}}{2 \omega_{1}} \sin (\chi-\phi), \\
& \dot{\phi}=-\delta \tilde{\omega}_{1}+\frac{3 \tilde{\gamma} a^{2}}{8 \omega_{1}}-\frac{\tilde{F}}{2 \omega_{1} a} \cos (\chi-\phi),
\end{aligned}
$$


where $\delta \tilde{\omega}_{1}=\omega_{F_{1}}-\tilde{\omega}_{1}$. For the steady-state response we set $\dot{a}=\dot{\phi}=0$, and obtain from Eqs. (17)-(18) the following well-known response curve of a forced Duffing resonator with nonlinear damping [32]

$$
a^{2}\left[\left(\delta \tilde{\omega}_{1}-\frac{3 \tilde{\gamma} a^{2}}{8 \omega_{1}}\right)^{2}+\left(\Gamma_{1 \mathrm{eff}}+\frac{\alpha_{2} \Gamma_{2} a^{2}}{4}\right)^{2}\right]=\left(\frac{\tilde{F}}{2 \omega_{1}}\right)^{2} .
$$

A standard stability analysis of this nonlinear response indicates the expected results for a forced Duffing resonator, with upper and lower stable branches of the response that are connected by the middle unstable branch of the response [26].

To illustrate the amplification of the primary resonator response due to the linear coupling of the resonators $G_{1}$, we use the following set of system parameters: $\omega_{1}=1, \omega_{2}=10, \Gamma_{1}=10^{-3}, \Gamma_{2}=1, F_{1}=10^{-3}, F_{2}=1, G_{1}=$ $4, G_{2}=0, \gamma=0.01$, which yields almost a threefold increase in the linear decay rate $\Gamma_{1 \mathrm{eff}} / \Gamma_{1}=2.63$, an $8 \%$ downward shift of the eigenfrequency $\Delta \omega_{1} / \omega_{1}=0.08$, and a forty times stronger driving force $\tilde{F} / F=41$. The numerically validated modified response curve is shown in Fig. 3 along with the response of the non-interacting primary resonator $(G=0)$. It is clear that the response is greatly enhanced due to the interaction with the secondary resonator. Specifically, the maximal amplitude of the response curve, $a_{\max }$, which satisfy the backbone curve equation $\omega_{\max }=\tilde{\omega}_{1}+3 \gamma a_{\max }^{2} /\left(8 \omega_{1}\right)$, is given by $a_{\max }=\tilde{F} /\left(2 \omega_{1} \Gamma_{1 \text { eff }}\right)$. Therefore, the maximal amplitude is increased by more than an order of magnitude, and the ratio between the maximal amplitude with and without coupling is given by $a_{\max } /\left(\left.a_{\max }\right|_{G=0}\right)=\Gamma_{1} \tilde{F} /\left(\Gamma_{1 \mathrm{eff}} F\right)=15.59$. Moreover, due to the variations in the eigenfrequency $\tilde{\omega}_{1}$ and in the maximal amplitude $a_{\max }$, the frequency of the maximal amplitude also increases by $14.34 \%$, and is explicitly given by $\omega_{\max }=\tilde{\omega}_{1}+3 \gamma \tilde{F}^{2} /\left(32 \omega_{1}^{3} \Gamma_{1 \mathrm{eff}}^{2}\right)$.

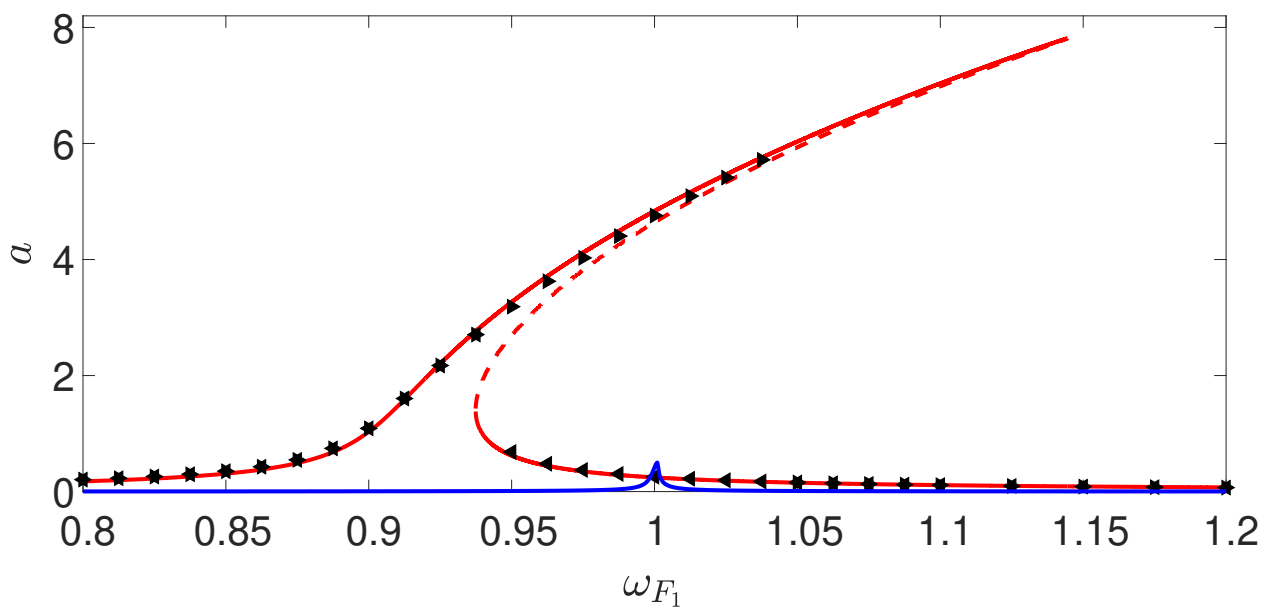

Fig. 3 Amplitude response curve of the primary resonator for the case of $\omega_{F_{2}}=\omega_{F_{1}}$. The red/blue curves are the responses that are obtained by analytically solving Eq. (19) with/without coupling to the secondary resonator, respectively. The solid/dashed curves indicate stable/unstable branches of the responses. The symbols $>$ and 4 indicate the sweep-up (increasing $\omega_{F_{1}}$ ) and sweepdown (decreasing $\omega_{F_{1}}$ ) values, respectively, of the numerically calculated amplitude of the primary resonator, which are obtained by performing numerical time integration of the original dynamical system, Eqs. (2)-(3). Note that the star symbol is the result of superimposition of oppositely facing triangles.

3.3 The case of $\omega_{F_{2}}=2 \omega_{F_{1}}$

In this case we have a combined parametric and directly driven Duffing resonator with nonlinear damping, and the polar evolution equations are given by

$$
\begin{aligned}
& \dot{a}=-\left(\Gamma_{1 \mathrm{eff}}+\frac{\alpha_{2} \Gamma_{2} a^{2}}{4}\right) a-\frac{F_{1}}{2 \omega_{1}} \sin (\phi-\theta)-\frac{k_{1} G_{2} F_{2}}{4 \omega_{1}} a \sin (2 \phi+\varphi), \\
& \dot{\phi}=-\delta \tilde{\omega}_{1}+\frac{3 \tilde{\gamma} a^{2}}{8 \omega_{1}}-\frac{F_{1}}{2 \omega_{1} a} \cos (\phi-\theta)-\frac{k_{1} G_{2} F_{2}}{4 \omega_{1}} \cos (2 \phi+\varphi) .
\end{aligned}
$$

For the steady-state response we set $\dot{a}=\dot{\phi}=0$, and obtain from Eqs. (20)-(21) the following equation of response curve

$$
a^{2}\left[\left(\delta \tilde{\omega}_{1}-\frac{3 \tilde{\gamma} a^{2}}{8 \omega_{1}}\right)^{2}+\left(\Gamma_{1 \mathrm{eff}}+\frac{\alpha_{2} \Gamma_{2} a^{2}}{4}\right)^{2}\right]=\frac{4 F_{1}^{2}+4 a k_{1} G_{2} F_{1} F_{2} \cos (\theta+\varphi+\phi)+a^{2} k_{1}^{2} G_{2}^{2} F_{2}^{2}}{16 \omega_{1}^{2}},
$$


and the following auxiliary equation for the phase $\phi$

$$
\frac{a k_{1} G_{2} F_{2} \cos (2 \phi+\varphi)+2 F_{1} \cos (\phi-\theta)}{a k_{1} G_{2} F_{2} \sin (2 \phi+\varphi)+2 F_{1} \sin (\phi-\theta)}=\frac{8 \omega_{1} \delta \tilde{\omega}_{1}-3 \tilde{\gamma} a^{2}}{8 \omega_{1} \Gamma_{1 \mathrm{eff}}+2 \omega_{1} \alpha_{2} \Gamma_{2} a^{2}} .
$$

As recently shown in Ref. [20], Eqs. (22)-(23) can generate intricate amplitude response curves that differ drastically from the standard directly driven Duffing resonator and include isolae, dual peaks, loops, and flat resonant peaks. However, we are interested here in a relatively simple response curve, which is amplified by the induced parametric excitation from the secondary resonator. To this end, we set $\theta=-\pi / 4[20]$ and use the following set of system parameters: $\omega_{1}=1, \omega_{2}=10, \Gamma_{1}=10^{-3}, \Gamma_{2}=1, F_{1}=10^{-3}, F_{2}=1, G_{1}=0, G_{2}=$ $5, \gamma=0.13$, which yields a drastic reduction in the Duffing nonlinearity $\tilde{\gamma} / \gamma=2.57 \times 10^{-2}(97.43 \%)$, a cubic nonlinear damping with a coefficient of $\alpha_{2} \Gamma_{2}=2.7 \times 10^{-3}$, and a parametric drive level of $k_{1} G_{2} F_{2}=0.05$. The numerically validated modified response curve is shown in Fig. 4 along with the response of the non-interacting primary resonator $(G=0)$. Clearly, the response of the primary resonator is amplified significantly due to the interaction with the secondary resonator. In particular, the maximal amplitude of the response curve, $a_{\max }$, is increased by almost an order of magnitude, where $a_{\max } /\left(\left.a_{\max }\right|_{G=0}\right)=8.41$, and its frequency $\omega_{\max }$ is barely changed by the interaction with secondary resonator (less than $1 \%$ variation).

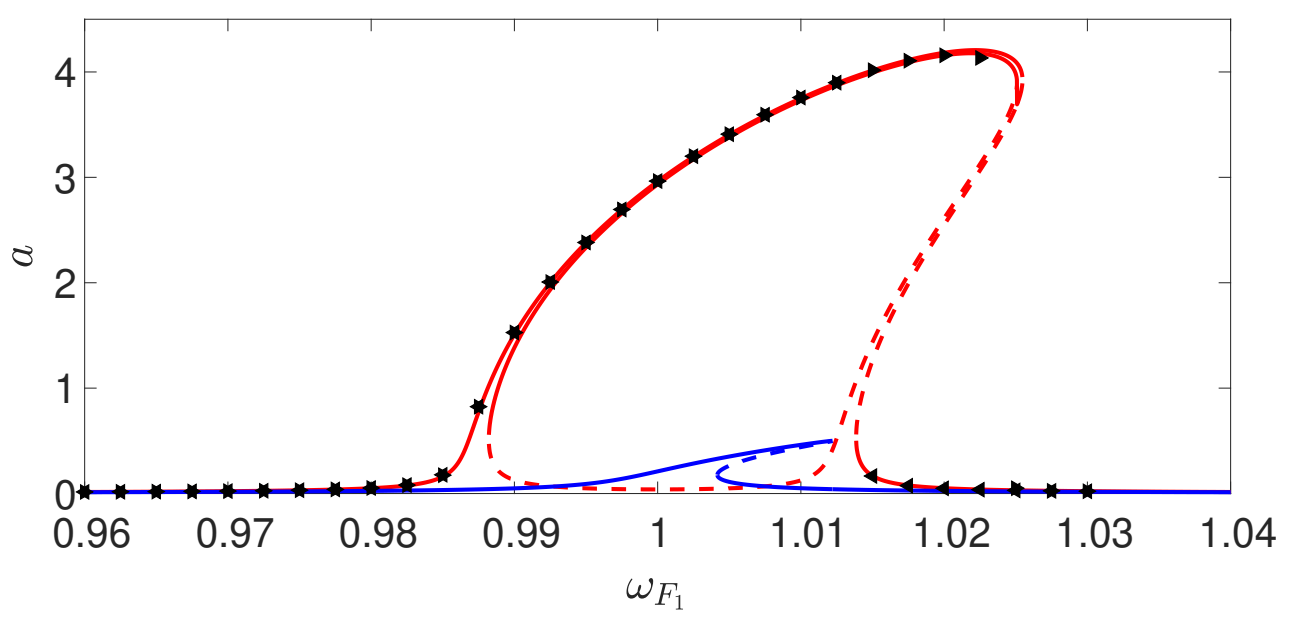

Fig. 4 Amplitude response curve of the primary resonator for the case of $\omega_{F_{2}}=2 \omega_{F_{1}}$. The red/blue curves are the response curves obtained by analytically solving Eqs. (22)-(23) with/without coupling to the secondary resonator, respectively. The solid/dashed curves indicate stable/unstable branches of the responses. The symbols $\boldsymbol{\downarrow}$ and $\varangle$ indicate the sweep-up (increasing $\left.\omega_{F_{1}}\right)$ and sweepdown (decreasing $\omega_{F_{1}}$ ) values, respectively, of the numerically calculated amplitude of the primary resonator, which are obtained by performing numerical time integration of the original dynamical system, Eqs. (2)-(3). Note that the star symbol is the result of superimposition of oppositely facing triangles.

\section{Closing remarks}

We analyzed the nonlinear interaction of mutually coupled driven resonators with significantly different decay rates. We considered a resonantly driven slowly decaying nonlinear (primary) resonator, which is nonlinearly coupled to a non-resonantly driven relatively fast decaying linear (secondary) resonator. We showed that when the drive frequency of the secondary resonator is close to either the eigenfrequency or twice the eigenfrequency of the primary resonator, the interaction between the resonators can be exploited to mechanically amplify the response of the primary resonator. In particular, the linear coupling between the resonators can be used to enhance the direct drive of the primary resonator when the drive frequency of the secondary resonator is close to the eigenfrequency of the primary resonator, and the quadratic coupling between the resonators can be used to parametrically pump the response of the primary resonator when the drive frequency of the secondary resonator is close to twice the eigenfrequency of the primary resonator.

We proposed a relatively simple amplification technique that can be analyzed theoretically in closed-form due to the large difference in the relaxation times of the resonators. Such systems of coupled resonators with large difference in their relaxation times are frequently encountered in coupling between MEMS (primary) and NEMS (secondary) resonators [29-31]. This amplification technique came from a straightforward generalization of a previous study on a similar undriven system [25], where it was shown that the linear and nonlinear characteristics of the primary resonator can be altered in a significant manner. Taken together the finding of the earlier analysis 
[25] and the current study, we conclude that such a driven dynamical system can be used to tailor a desired response curve of the primary resonator with prescribed linear and nonlinear characteristics, and a specified amplification.

In future studies we plan to develop methods for designing and tuning the coupling between the resonators in a mechanical manner. Mechanical realization of the coupling between the resonators can help to reduce the noise in the system by avoiding the use of inherently noisy electrical circuitry. To this end, we will use tools of structural optimization such as those described in Ref. [33], which will guide us in adjusting the coupling terms in our reduced-order models of the resonators.

\section{Funding:}

The results described herein are based on previous efforts that were carried out over the last couple of years with Prof. Steven W. Shaw and Prof. Mark I. Dykman. The work of the authors is supported by the United States - Israel Binational Science Foundation (BSF) under Grant No. 2018041, and by the Pearlstone Center of Aeronautical Engineering Studies at Ben-Gurion University of the Negev.

\section{Conflict of Interest:}

The authors declare that they have no conflict of interest.

\section{References}

1. M. LaHaye, O. Buu, B. Camarota, K. Schwab, Science 304(5667), 74 (2004)

2. Y.T. Yang, C. Callegari, X. Feng, K.L. Ekinci, M.L. Roukes, Nano letters 6(4), 583 (2006)

3. D. Rugar, R. Budakian, H. Mamin, B. Chui, Nature 430(6997), 329 (2004)

4. P. Kim, B. Hauer, C. Doolin, F. Souris, J. Davis, Nature communications 7(1), 1 (2016)

5. A.N. Cleland, M.L. Roukes, Nature 392(6672), 160 (1998)

6. J.F. Rhoads, N.J. Miller, S.W. Shaw, B.F. Feeny, Journal of Vibration and Acoustics 130(6) (2008)

7. J. Suh, M.D. LaHaye, P.M. Echternach, K.C. Schwab, M.L. Roukes, Nano letters 10(10), 3990 (2010)

8. J.F. Rhoads, S.W. Shaw, Applied Physics Letters 96(23), 234101 (2010)

9. R. Karabalin, R. Lifshitz, M. Cross, M. Matheny, S. Masmanidis, M. Roukes, Physical review letters 106(9), 094102 (2011)

10. N.J. Miller, S.W. Shaw, Journal of dynamic systems, measurement, and control 134(2) (2012)

11. Z. Yie, N.J. Miller, S.W. Shaw, K.L. Turner, Journal of Micromechanics and Microengineering 22(3), 035004 (2012)

12. S. Ilyas, N. Jaber, M.I. Younis, Journal of Microelectromechanical Systems 25(5), 916 (2016)

13. P.M. Polunin, S.W. Shaw, International Journal of Non-Linear Mechanics 94, 300 (2017)

14. S. Ilyas, N. Jaber, M.I. Younis, IEEE Transactions on Electron Devices 64(6), 2659 (2017)

15. M. Hasan, F.M. Alsaleem, N. Jaber, M.A.A. Hafiz, M.I. Younis, Aip Advances 8(1), 015312 (2018)

16. J.M. Miller, N.E. Bousse, D.D. Shin, H.K. Kwon, T.W. Kenny, in 2019 20th International Conference on Solid-State Sensors, Actuators and Microsystems \& Eurosensors XXXIII (TRANSDUCERS \& EUROSENSORS XXXIII) (IEEE, 2019), pp. 881884

17. S. Iqbal, A. Malik, Sensors and Actuators A: Physical 300, 111666 (2019)

18. X. Zheng, H. Wu, Y. Lin, Z. Ma, Z. Jin, Journal of Micromechanics and Microengineering 30(4), 045010 (2020)

19. D. Bothner, S. Yanai, A. Iniguez-Rabago, M. Yuan, Y.M. Blanter, G.A. Steele, Nature communications 11(1), 1 (2020)

20. D. Li, S.W. Shaw, Nonlinear Dynamics 102(4), 2433 (2020)

21. J.H. Scofield, American journal of physics 62(2), 129 (1994)

22. G.C. Hsieh, J.C. Hung, IEEE Transactions on industrial electronics 43(6), 609 (1996)

23. W. Mumford, Proceedings of the IRE 48(5), 848 (1960)

24. M. Metcalfe, Applied Physics Reviews 1(3), 031105 (2014)

25. O. Shoshani, M.I. Dykman, S.W. Shaw, Nonlinear Dynamics 99(1), 433 (2020)

26. A.H. Nayfeh, D.T. Mook, Nonlinear oscillations (John Wiley \& Sons, 2008)

27. J. Guckenheimer, P.J. Holmes, Nonlinear oscillations, dynamical systems, and bifurcations of vector fields, vol. 42 (Springer Science \& Business Media, 2013)

28. O. Shoshani, S.W. Shaw, M.I. Dykman, Scientific reports 7(1), 18091 (2017)

29. I. Mahboob, N. Perrissin, K. Nishiguchi, D. Hatanaka, Y. Okazaki, A. Fujiwara, H. Yamaguchi, Nano letters 15(4), 2312 (2015)

30. F. Sun, X. Dong, J. Zou, M.I. Dykman, H.B. Chan, Nature communications 7(1), 1 (2016)

31. X. Dong, M.I. Dykman, H.B. Chan, Nature communications 9(1), 1 (2018)

32. R. Lifshitz, M. Cross, Review of nonlinear dynamics and complexity 1, 1 (2008)

33. S. Dou, B.S. Strachan, S.W. Shaw, J.S. Jensen, Philosophical Transactions of the Royal Society A: Mathematical, Physical and Engineering Sciences 373(2051), 20140408 (2015) 
Figures

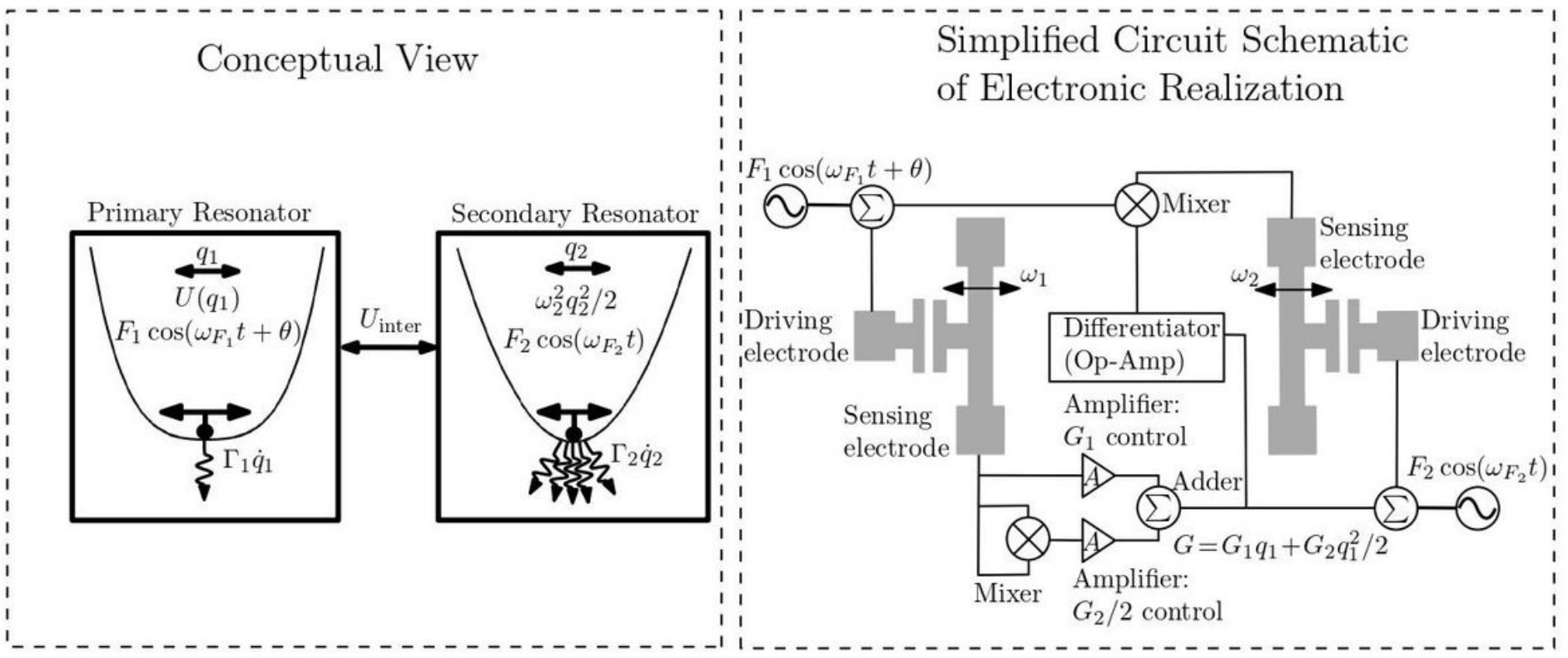

Figure 1

Please see the Manuscript PDF file for the complete figure caption
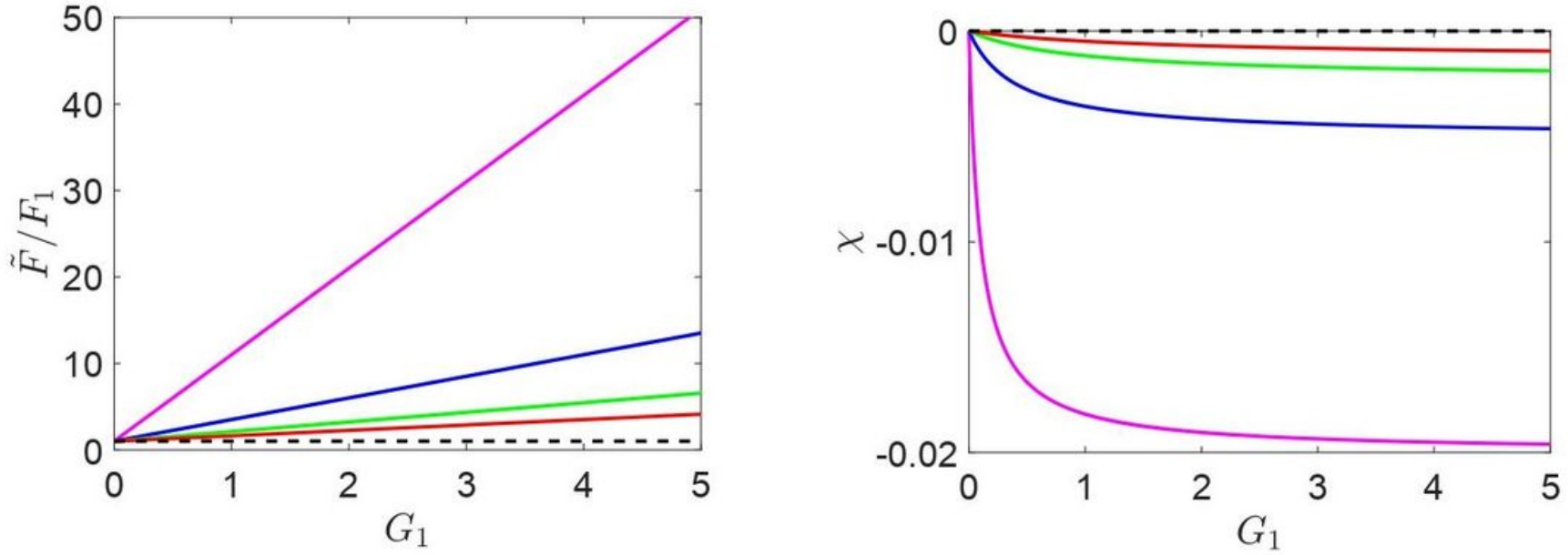

Figure 2

Please see the Manuscript PDF file for the complete figure caption 


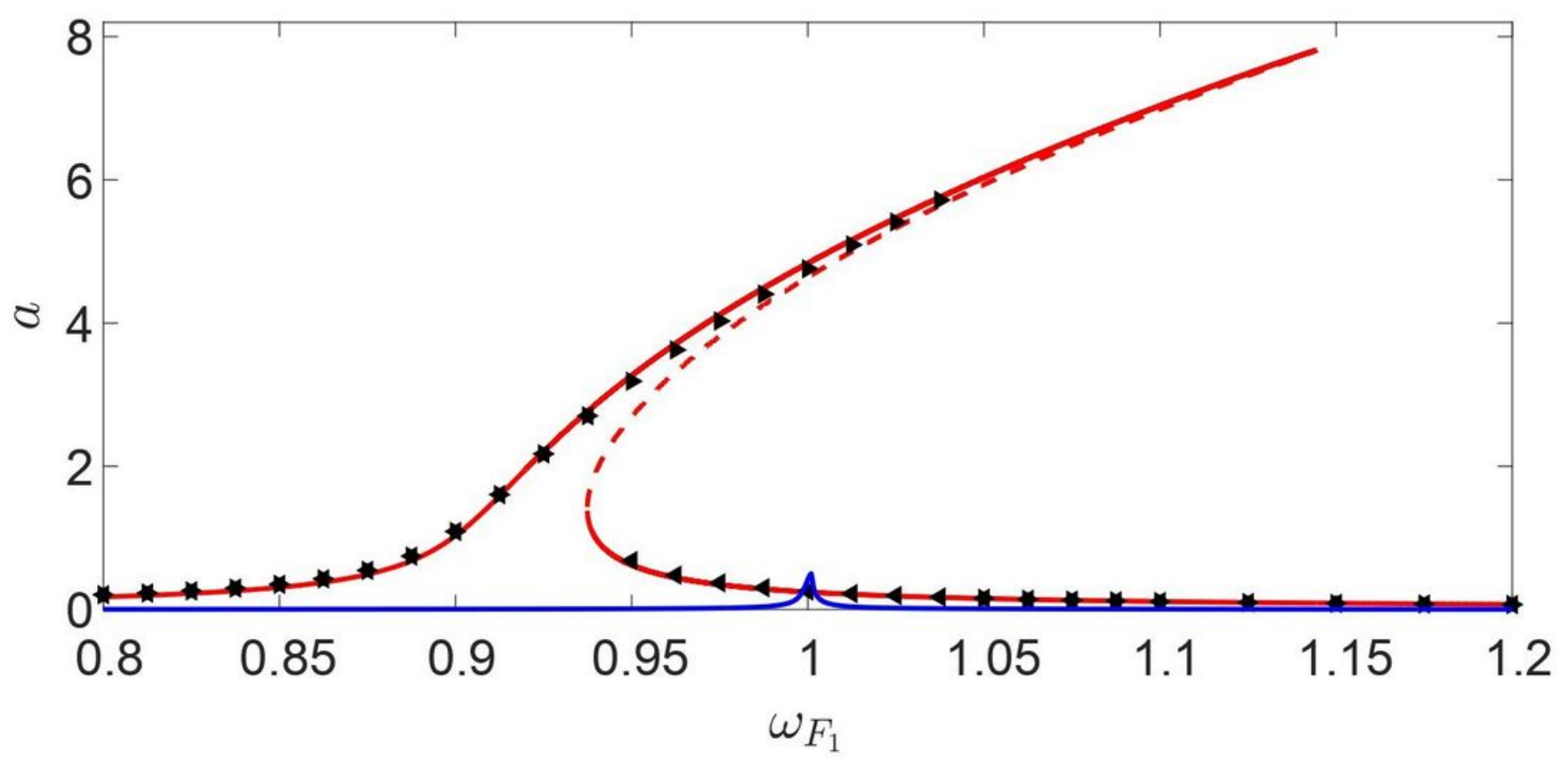

Figure 3

Please see the Manuscript PDF file for the complete figure caption

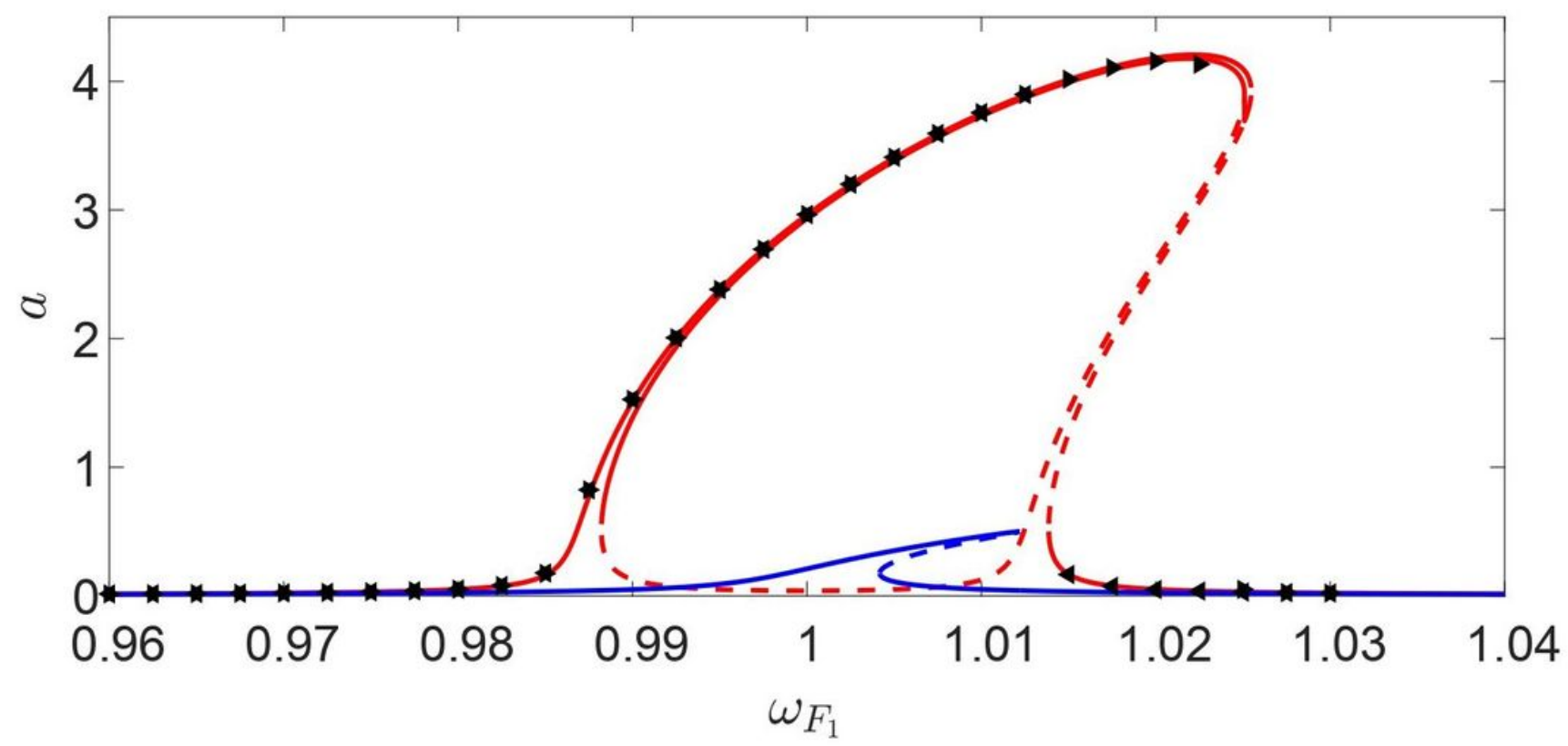

Figure 4

Please see the Manuscript PDF file for the complete figure caption 